\title{
Variation in hospital morbidity in the male workforce of Western Australia
}

\author{
V P WADDELL, ' C D J HOLMAN,' B K ARMSTRONG, ${ }^{2}$ J C McNULTY,' \\ P PSAILA-SAVONA'
}

From the Public Health and Scientific Support Services, ${ }^{1}$ Health Department of Western Australia, Perth 6000 , and NH \& MRC Research Unit in Epidemiology and Preventive Medicine, ${ }^{2}$ Department of Medicine, University of Western Australia, Perth, Western Australia

ABSTRACT The associations of hospital morbidity with occupation were studied in Western Australian men of working age in 1981-2. Data on hospital morbidity were derived from a population based system that covered all short stays in hospitals in the state. Occupations were grouped into 12 major categories and conditions were coded using the International Classification of Diseases. Armed services personnel had the highest overall rate of hospital admissions, followed by transport and communications workers and by administrative and managerial workers. Injuries were the most common cause of referral to hospital. Four occupational groups, farmers and allied workers, miners and quarrymen, transport and communications workers, and craftsmen, production workers, and labourers were often associated with injury. Diseases of the musculoskeletal system and connective tissue were the second most common cause for referral and were increased in transport and communications workers, and in craftsmen, production workers, and labourers. Several other associations between causes of hospital admission and major occupational groups were observed, including associations of circulatory system diseases with professional and technical work and with administrative and managerial work. The excess of hospital admissions due to factors associated with occupation was estimated to be 12665 admissions a year or $24.9 \%$ of the total in working men.

Whereas studies of the relation of mortality to occupational factors are commonplace, there are few published data showing the effects of occupation on rates of admission to hospital. There are probably several reasons for this. Death is a comparatively rare event and an important one. Deaths are easily defined and are routinely documented and enumerated in most countries. This contrasts with the infrequency of population based data on hospital morbidity. Moreover, compared with mortality studies, analyses of hospital morbidity are both difficult to perform and difficult to interpret correctly. These difficulties are due to the potential for variation in the criteria for hospital admission and in the number of admissions per illness episode.

Western Australia occupies the western third of the Australian continent and has a population of $1.3 \mathrm{~m}$ people, $71 \%$ of whom live in the capital city, Perth. Most of the population is of European origin, the aboriginal people and other races comprising around $2.5 \%$ each. The major industries are mining (iron ore,

Accepted 26 January 1987 coal, oil, gold, diamonds, uranium, and formerly asbestos) and farming (wheat, sheep, and cattle). Since 1971 data on all admissions to short stay hospitals in Western Australia have been entered into a population based hospital morbidity data system (HMDS). Among the demographic variables recorded has been the occupation of each patient current at the time of admission to hospital. In this paper we summarise the differences in rates of admission to hospital in men aged 15 to 64 according to occupational group. A more detailed report of the study has been published by the Health Department of Western Australia. ${ }^{1}$

\section{Methods}

SOURCES OF MORBIDITY AND POPULATION DATA

The numerators for analysis, numbers of short stay hospital discharges in 1981-2, were derived from the Western Australian HMDS. There was a total of 619240 short stay hospital discharges in the two years, of which 101548 were of employed men aged 15 to 64 . Males under 15 or over 64 as well as all females, were 
excluded from analysis in view of the high proportions whose occupation was described as student, housewife, retired, or pensioner.

The forms used by the HMDS in 1981-2 were in two parts. The first recorded demographic details such as sex, date of birth, usual place of residence, and present occupation, and was completed on admission to hospital by an admitting clerk; the second was completed on leaving and consisted of information about the morbid conditions treated and the surgical or medical procedures performed in hospital. The forms allowed for the recording of the "principal condition treated," the "external cause" (where different), and a variable number of "other conditions present" to be stated. The principal condition treated and external cause were the morbidity items selected for this analysis.

Populations of Western Australian men according to age and major occupational group were derived from the Australian Bureau of Statistics' census of 30 June 1981. Intercensal population estimates for 1982 were available by age and sex but not by occupation. To obtain denominators by occupational group for 1982 , the occupational distribution in 1981 was applied to the 1982 population estimates. Overall, it was estimated that there were 881794 person-years lived by men aged 15-64 in Western Australia in 19812 , of which $76.8 \%$ were lived by employed men, $20.3 \%$ by men not in the workforce, and $3.0 \%$ by men whose occupation was inadequately described or not stated. The largest major occupational group was craftsmen, production process workers, and labourers, contributing 266838 man-years. The smallest group was members of the armed services, with 5859 man-years (see table 1).

CLASSIFICATIONS OF OCCUPATION AND DISEASE

In the HMDS occupation was coded according to a

Table 1 Populations of Western Australian men aged 15-64 in 1981-2 according to major occupational group

\begin{tabular}{clr}
\hline & Major occupational group & Person-years \\
\hline 0 & Professional, technical, and related workers & 81977 \\
1 & Administrative, executive, and managerial & 66922 \\
& $\quad$ workers & 50762 \\
2 & Clerical workers & 40400 \\
3 & Sales workers & 67130 \\
4 & Farmers, fishermen, hunters, timbergetters, & 14145 \\
5 & and related workers & 48372 \\
6 & Winers, quarrymen, and related workers & 266838 \\
$7 / 8$ & Craftsmen, production process workers, & 34473 \\
9 & and labourers & 5859 \\
10 & Service, sport, and recreation workers & 26268 \\
11 & Occupers of armed services & 178648 \\
& not stated & 881794 \\
\hline
\end{tabular}

two digit system published by the Australian Bureau of Statistics and consisting of 12 major codes and 94 minor codes. In the census occupation was coded using a three digit system incorporating the same 12 major codes but having 865 minor codes. The 12 major codes have been used in tabulating our results. Little emphasis was placed on major occupational groups 11 (occupation inadequately described or not stated) and 12 (people not in the workforce) as our major aim was to investigate associations between occupation and disease.

The classification of illnesses and injuries in the HMDS was based on the ninth revision of the International Classification of Diseases (ICD-9). Analysis of the morbidity data was performed by grouping the ICD codes of the principal condition in two ways, firstly, by ICD-9 chapters and secondly by the ICD-9 basic tabulation list. There are 17 ICD-9 chapters and 67 items relating to disease or injury type in the basic tabulation list. The two supplementary classifications of ICD-9 (E-codes and V-codes) allow additional information to be recorded. The E-codes relate to the external causes of injury and poisoning. The V-codes are used for situations that cannot be classified using the chapters-for example, screening procedures, fertility control, and need for isolation. The basic tabulation list includes 10 items for the Ecodes and one for the V-codes. Chapters XI, XIV, and $\mathrm{XV}$ relating to pregnancy and childbirth, congenital anomalies, and perinatal conditions were irrelevant to the study and were, therefore, excluded.

\section{METHODS OF ANALYSIS}

The measures calculated from the data reported in the tables are precision based rate ratios (RR) and odds ratios (OR) with associated $95 \%$ confidence intervals and null $\mathrm{p}$ values. The methods of calculation were those described by Kleinbaum et al, ${ }^{2}$ with the exception that in calculating the $95 \%$ confidence intervals of rate ratios we used a formula for the variance of the natural log of the stratum specific estimates based on the Poisson distribution. ${ }^{3}$ This latter approach appeared more robust than the alternative formulation described by Kleinbaum et al. ${ }^{2}$

Thus in the ith age group,

$$
\begin{aligned}
\mathrm{RR}_{\mathrm{i}} & =\frac{\mathrm{d}_{\mathrm{li}}}{\mathrm{PT}_{\mathrm{li}}} / \frac{\mathrm{d}_{\mathrm{oi}}}{\mathbf{P T}_{\mathrm{oi}}} \\
\text { and } & \mathrm{OR}_{\mathrm{i}}=\frac{\mathrm{d}_{\mathrm{li}}}{\mathrm{c}_{\mathrm{li}}} / \frac{\mathrm{d}_{\mathrm{oi}}}{\mathrm{c}_{\mathrm{oi}}}
\end{aligned}
$$

where $d_{1 i}$ and $d_{o i}$ were the numbers of hospital discharges in the exposed and non-exposed; $\mathrm{PT}_{\mathrm{li}}$ and $\mathrm{PT}_{\mathrm{oi}}$ were the corresponding amounts of person time based on census data; and $c_{l i}$ and $c_{o i}$ were the numbers of "referent events" in the exposed and non-exposed (see below). The pooled estimates of RR and OR were obtained from, 


$$
\begin{array}{r}
\mathrm{RR}=\exp \left|\frac{\sum \mathbf{W}_{\mathbf{R R i}} \ln \left(\mathrm{RR}_{\mathrm{i}}\right)}{\sum \mathbf{W}_{\mathrm{RRi}}}\right| \\
\text { and } \\
\mathrm{OR}=\exp \left|\frac{\sum \mathbf{W}_{\mathrm{ORi}} \ln \left(\mathrm{RR}_{\mathrm{i}}\right)}{\sum \mathbf{W}_{\mathrm{ORi}}}\right|
\end{array}
$$

where the $\mathrm{W}_{\mathrm{RRi}}$ and $\mathrm{W}_{\mathrm{ORi}}$ were the precision based weights equal to the reciprocals of the variances of $R_{R_{i}}$ and $\mathrm{OR}_{\mathrm{i} \cdot}{ }^{23}$

Some aspects of our study design and analysis departed from common practice. In the analysis exposure contrasts were always formed between a major occupational group (the "exposed") and all other subjects in the male workforce (the "non- exposed"). It provides for a purer contrast than if the general population is used as the non-exposed group, because both exposed subjects and those who have left the workforce because of sickness are excluded.

Explanation of the use of the OR in occupational studies, including argument as to why the OR is preferred to the proportional morbidity or mortality ratio (PMR) has been outlined by Miettinen and Wang. ${ }^{45}$ The design concept is essentially that of a case-control study and leads naturally to an estimate of the morbidity and mortality rate ratio arising from the $O R$ as the measure of effect. In the investigation of a specific disease occupation relation by use of the OR, much attention would normally be given to the

\begin{tabular}{|c|c|c|c|c|c|c|c|c|}
\hline \multirow{2}{*}{\multicolumn{2}{|c|}{ Major occupational group }} & \multicolumn{3}{|c|}{ Rate ratio } & \multicolumn{3}{|c|}{ Odds ratio } & \multirow{2}{*}{$\begin{array}{l}\text { Credibility } \\
\text { score } \\
(\text { Range 0-6) }\end{array}$} \\
\hline & & $\overline{E s t}$ & $95 \% C I$ & Null $p$ value & $\overline{E s t}$ & $95 \% C I$ & Null $p$ value & \\
\hline $\begin{array}{l}4 \\
9\end{array}$ & $\begin{array}{l}\text { Chapter I: Infectious and parasitic diseases } \\
\text { Farmers, fishermen, hunters, timbergetters } \\
\text { Service, sport, and recreation workers }\end{array}$ & $\begin{array}{l}1.41 \\
1.36\end{array}$ & $\begin{array}{l}1 \cdot 22-1.63 \\
1 \cdot 12-1.66\end{array}$ & $\begin{array}{l}10^{-6} \\
0.007\end{array}$ & $\begin{array}{l}1 \cdot 20 \\
1 \cdot 32\end{array}$ & $\begin{array}{l}1.04-1.39 \\
1.08-1.61\end{array}$ & $\begin{array}{l}0.023 \\
0.019\end{array}$ & $\begin{array}{l}3 \\
3\end{array}$ \\
\hline $\begin{array}{r}0 \\
10\end{array}$ & $\begin{array}{l}\text { Chapter II: Neoplasms } \\
\text { Professional, technical, and related workers } \\
\text { Members of armed services }\end{array}$ & $\begin{array}{l}1 \cdot 25 \\
3.99\end{array}$ & $\begin{array}{l}1 \cdot 13-1 \cdot 38 \\
2 \cdot 80-5 \cdot 70\end{array}$ & $\begin{array}{l}10^{-5} \\
10^{-6}\end{array}$ & $\begin{array}{l}1 \cdot 56 \\
2 \cdot 51\end{array}$ & $\begin{array}{l}1.41-1.73 \\
1.74-3.63\end{array}$ & $\begin{array}{l}<10^{-8} \\
0.002\end{array}$ & $\begin{array}{l}3 \\
3\end{array}$ \\
\hline 9 & $\begin{array}{l}\text { Chapter III: Endocrine, nutritional, and } \\
\text { metabolic diseases and immunity disorders } \\
\text { Service, sport, and recreation workers }\end{array}$ & $1 \cdot 69$ & $1 \cdot 28-2 \cdot 23$ & 0.001 & $9 \cdot 24$ & $7 \cdot 89-10 \cdot 82$ & $<10^{-8}$ & 2 \\
\hline 9 & $\begin{array}{l}\text { Chapter V: Mental disorders } \\
\text { Service, sport, and recreation workers }\end{array}$ & $1 \cdot 59$ & $1 \cdot 38-1 \cdot 83$ & $10^{-8}$ & $1 \cdot 45$ & $1.26-1.68$ & $10^{-6}$ & 2 \\
\hline 4 & $\begin{array}{l}\text { Chapter VI: Diseases of the nervous system } \\
\text { and sense organs } \\
\text { Farmers, fishermen, hunters, timbergetters }\end{array}$ & $1 \cdot 31$ & $1 \cdot 19-1 \cdot 43$ & $10^{-8}$ & $1 \cdot 16$ & $1.06-1.28$ & 0.002 & 0 \\
\hline \multirow{3}{*}{$\begin{array}{l}0 \\
1\end{array}$} & \multirow{3}{*}{$\begin{array}{l}\text { Chapter VII: Diseases of the circulatory } \\
\text { system } \\
\text { Professional, technical, and related workers } \\
\text { Administrative, executive, and managerial } \\
\text { workers }\end{array}$} & & & & & & & \\
\hline & & $1 \cdot 20$ & $1 \cdot 13-1 \cdot 28$ & $10^{-5}$ & 1.48 & $1.39-1.59$ & $<10^{-8}$ & 2 \\
\hline & & $1 \cdot 22$ & $1 \cdot 15-1 \cdot 30$ & $<10^{-8}$ & $1 \cdot 13$ & $1.06-1.21$ & 0.001 & 3 \\
\hline 1 & $\begin{array}{l}\text { Chapter VIII: Diseases of the respiratory } \\
\text { system } \\
\text { Administrative, executive, and managerial } \\
\text { workers }\end{array}$ & $1 \cdot 21$ & $1 \cdot 11-1 \cdot 31$ & $10^{-5}$ & $1 \cdot 15$ & $1.05-1.25$ & 0.005 & 2 \\
\hline 1 & $\begin{array}{l}\text { Chapter IX: Diseases of the digestive system } \\
\text { Administrative, executive, and managerial } \\
\text { workers }\end{array}$ & $1 \cdot 21$ & $1 \cdot 14-1 \cdot 28$ & $<10^{-8}$ & $1 \cdot 14$ & $1.07-1.21$ & $10^{-5}$ & 3 \\
\hline \multirow{3}{*}{$\begin{array}{l}6 \\
7 / 8\end{array}$} & $\begin{array}{l}\text { Chapter XIII: Diseases of the musculoskeletal } \\
\text { system and connective tissue }\end{array}$ & & & & & & & \\
\hline & $\begin{array}{l}\text { Workers in transport and communications } \\
\text { Craftsmen, production process workers, }\end{array}$ & $1 \cdot 37$ & $1 \cdot 29-1 \cdot 45$ & $<10^{-8}$ & $1 \cdot 10$ & $1 \cdot 03-1 \cdot 17$ & 0.011 & 5 \\
\hline & and labourers & $1 \cdot 25$ & $1 \cdot 21-1 \cdot 30$ & $<10^{-8}$ & 1.91 & $1 \cdot 15-1 \cdot 24$ & $<10^{-8}$ & 4 \\
\hline $\begin{array}{l}1 \\
4 \\
9\end{array}$ & $\begin{array}{l}\text { Chapter XVI: Symptoms, signs, and } \\
\text { ill defined conditions } \\
\text { Administrative, executive, and managerial } \\
\text { workers } \\
\text { Farmers, fishermen, hunters, timbergetters } \\
\text { Service, sport, and recreation workers }\end{array}$ & $\begin{array}{l}1 \cdot 19 \\
1 \cdot 48 \\
1 \cdot 18\end{array}$ & $\begin{array}{l}1 \cdot 11-1 \cdot 27 \\
1 \cdot 39-1 \cdot 58 \\
1 \cdot 08-1 \cdot 30\end{array}$ & $\begin{array}{c}10^{-6} \\
<10^{-8} \\
0.002\end{array}$ & $\begin{array}{l}1 \cdot 11 \\
1 \cdot 33 \\
1 \cdot 12\end{array}$ & $\begin{array}{l}1.04-1.19 \\
1.24-1.42 \\
1.02-1.23\end{array}$ & $\begin{array}{c}0.005 \\
<10^{-8} \\
0.038\end{array}$ & $\begin{array}{l}0 \\
2 \\
0\end{array}$ \\
\hline \multirow[t]{2}{*}{$\begin{array}{l}4 \\
5 \\
7 / 8\end{array}$} & $\begin{array}{l}\text { Chapter XVII: Injury and poisoning } \\
\text { Farmers, fishermen, hunters, timbergetters } \\
\text { Miners, quarrymen, and related workers }\end{array}$ & $\begin{array}{l}1.58 \\
1.65\end{array}$ & $\begin{array}{l}1 \cdot 52-1 \cdot 64 \\
1.53-1 \cdot 78\end{array}$ & $\begin{array}{l}<10^{-8} \\
<10^{-8}\end{array}$ & $\begin{array}{l}1 \cdot 34 \\
1 \cdot 56\end{array}$ & $\begin{array}{l}1.28-1.40 \\
1.43-1.70\end{array}$ & $\begin{array}{l}<10^{-8} \\
<10^{-8}\end{array}$ & $\begin{array}{l}6 \\
6\end{array}$ \\
\hline & and labourers & 1.25 & $1 \cdot 22-1 \cdot 29$ & $<10^{-8}$ & $1 \cdot 17$ & $1 \cdot 13-1.21$ & $<10^{-8}$ & 3 \\
\hline
\end{tabular}

Table 2 Empirical associations and credibility scores: ICD-9 chapters 


\begin{tabular}{|c|c|c|c|c|c|c|c|c|}
\hline \multirow{2}{*}{\multicolumn{2}{|c|}{ Major occupational group }} & \multicolumn{3}{|c|}{ Rate ratio } & \multicolumn{3}{|c|}{ Odds ratio } & \multirow{2}{*}{$\begin{array}{l}\text { Credibility } \\
\text { score } \\
\text { (Range 0-6) }\end{array}$} \\
\hline & & $\overline{E s t}$ & $95 \% \mathrm{CI}$ & Null $p$ value & $\overline{\text { Est }}$ & $95 \% \mathrm{CI}$ & Null p valuc & \\
\hline \multirow[b]{2}{*}{$\begin{array}{l}4 \\
6\end{array}$} & \multicolumn{8}{|l|}{ (01) Intestinal infectious diseases } \\
\hline & $\begin{array}{l}\text { Farmers, fishermen, hunters, and timbergetters } \\
\text { Workers in transport and communications }\end{array}$ & $\begin{array}{l}2 \cdot 23 \\
1.89\end{array}$ & $\begin{array}{l}1 \cdot 17-2 \cdot 86 \\
1 \cdot 40-2 \cdot 55\end{array}$ & $\begin{array}{r}<10^{-8} \\
10^{-4}\end{array}$ & $\begin{array}{l}1.88 \\
1.52\end{array}$ & $\begin{array}{l}1 \cdot 46-2 \cdot 41 \\
1 \cdot 12-2 \cdot 05\end{array}$ & $\begin{array}{c}10^{-6} \\
0 \cdot 017\end{array}$ & 1 \\
\hline 9 & $\begin{array}{l}\text { Service, sport, and recreation workers } \\
\text { (11) Malignant neoplasms of bone, connective } \\
\text { tissue, skin, and breast }\end{array}$ & 1.65 & $1 \cdot 28-2 \cdot 11$ & $10^{-4}$ & $1 \cdot 58$ & $1 \cdot 23-2 \cdot 04$ & 0.001 & $\mathbf{0}$ \\
\hline $\begin{array}{l}\mathbf{0} \\
2\end{array}$ & $\begin{array}{l}\text { Professional, technical, and related workers } \\
\text { Clerical workers } \\
\text { (13) Malignant neoplasm of other and unspecified }\end{array}$ & 1.63 & $\begin{array}{l}1.34-2.00 \\
1 \cdot 18-1.96\end{array}$ & $\begin{array}{l}10^{-5} \\
0.009\end{array}$ & $\begin{array}{l}2.08 \\
1 \cdot 84\end{array}$ & $\begin{array}{l}1 \cdot 70-2 \cdot 56 \\
1 \cdot 42-2 \cdot 38\end{array}$ & $\begin{array}{r}<10^{-8} \\
10^{-5}\end{array}$ & $\begin{array}{l}2 \\
2\end{array}$ \\
\hline 4 & $\begin{array}{l}\text { Farmers, fishermen, hunters, and timbergetters } \\
\text { (15) Benign neoplasms }\end{array}$ & 1.57 & $1 \cdot 20-2 \cdot 04$ & 0.004 & 1.46 & $1 \cdot 12-1 \cdot 90$ & 0.020 & 1 \\
\hline \multirow{2}{*}{$\begin{array}{l}0 \\
1\end{array}$} & Professional, technical, and related workers & $1 \cdot 33$ & $1 \cdot 07-1 \cdot 66$ & 0.018 & 1.73 & $1 \cdot 39-2 \cdot 16$ & $10^{-6}$ & 0 \\
\hline & $\begin{array}{l}\text { workers } \\
\text { (18) Endocrine and metabolic diseases, immunity } \\
\text { disorders }\end{array}$ & 1.46 & $1 \cdot 17-1 \cdot 82$ & 0.002 & 1.38 & $1 \cdot 11-1 \cdot 73$ & 0.007 & 0 \\
\hline 9 & $\begin{array}{l}\text { Service, sport, and recreation workers } \\
\text { (22) Diseases of the nervous system }\end{array}$ & $1 \cdot 73$ & $1 \cdot 31-2 \cdot 28$ & $10^{-4}$ & $1 \cdot 71$ & $1 \cdot 29-2 \cdot 25$ & 0.001 & 3 \\
\hline $\begin{array}{l}4 \\
9\end{array}$ & $\begin{array}{l}\text { Farmers, fishermen, hunters, and timbergetters } \\
\text { Service, sport, and recreation workers } \\
\text { (23) Disorders of the eye and adnexa }\end{array}$ & $\begin{array}{l}1.41 \\
1.54\end{array}$ & $\begin{array}{l}1.21-1.63 \\
1.27-1.86\end{array}$ & $\begin{array}{l}10^{-4} \\
10^{-5}\end{array}$ & $\begin{array}{l}1 \cdot 24 \\
1 \cdot 48\end{array}$ & $\begin{array}{l}1.07-1.45 \\
1 \cdot 22-1.80\end{array}$ & $\begin{array}{l}0.018 \\
0.001\end{array}$ & $\begin{array}{l}\mathbf{0} \\
\mathbf{0}\end{array}$ \\
\hline 4 & $\begin{array}{l}\text { Farmers, fishermen, hunters, and timbergetters } \\
\text { (26) Hypertensive diseases }\end{array}$ & 1.59 & $1 \cdot 40-1.81$ & $<10^{-8}$ & 1.45 & $1 \cdot 27-1 \cdot 65$ & $10^{-8}$ & 2 \\
\hline \multirow{2}{*}{$\begin{array}{l}0 \\
7 / 8\end{array}$} & Professional, technical, and related workers & $3 \cdot 55$ & $2 \cdot 97-4 \cdot 24$ & $<10^{-8}$ & $4 \cdot 82$ & $4 \cdot 02-5 \cdot 78$ & $<10^{-8}$ & 一* \\
\hline & $\begin{array}{l}\text { Craftsmen, production process workers, } \\
\text { and labourers } \\
\text { (27) Ischaemic heart disease }\end{array}$ & 1.62 & $1.42-1.84$ & $<10^{-8}$ & 1.53 & $1 \cdot 34-1 \cdot 74$ & $<10^{-8}$ & 1 \\
\hline 1 & $\begin{array}{l}\text { Administrative, executive, and managerial } \\
\text { workers }\end{array}$ & 1.51 & $1.37-1.66$ & $<10^{-8}$ & 1.41 & $1 \cdot 28-1 \cdot 59$ & $<10^{-8}$ & 4 \\
\hline 2 & $\begin{array}{l}\text { Clerical workers } \\
(28) \text { Diseases of pulmonary circulation and other } \\
\text { forms of heart disease }\end{array}$ & $1 \cdot 22$ & $1.06-1.39$ & 0.015 & 1.47 & $1.27-1.69$ & $10^{-7}$ & 2 \\
\hline 1 & $\begin{array}{l}\text { Administrative, executive, and managerial } \\
\text { workers }\end{array}$ & 1.43 & $1 \cdot 21-1.69$ & $10^{-5}$ & 1.38 & $1 \cdot 12-1 \cdot 57$ & 1.001 & 2 \\
\hline 9 & $\begin{array}{l}\text { Service, sport, and recreation workers } \\
\text { (30) Other diseases of the circulatory system }\end{array}$ & $1 \cdot 39$ & $1 \cdot 10-1 \cdot 76$ & 0.035 & 1.39 & $1.09-1.76$ & 0.044 & 1 \\
\hline 1 & $\begin{array}{l}\text { Administrative, executive, and managerial } \\
\text { workers }\end{array}$ & $1 \cdot 24$ & $1 \cdot 11-1 \cdot 37$ & 0.001 & $1 \cdot 18$ & $1.06-1.32$ & 0.016 & 1 \\
\hline $\begin{array}{l}2 \\
9\end{array}$ & $\begin{array}{l}\text { Clerical workers } \\
\text { Service, sport, and recreation workers } \\
\text { (31) Diseases of the upper respiratory tract }\end{array}$ & $\begin{array}{l}1 \cdot 18 \\
1 \cdot 43\end{array}$ & $\begin{array}{l}1.03-1.35 \\
1.24-1.64\end{array}$ & $\begin{array}{l}0.039 \\
10^{-6}\end{array}$ & $\begin{array}{l}1.47 \\
1.36\end{array}$ & $\begin{array}{l}1.29-1.69 \\
1 \cdot 18-1.57\end{array}$ & $\begin{array}{l}10^{-7} \\
10^{-5}\end{array}$ & $\begin{array}{l}2 \\
0\end{array}$ \\
\hline 1 & $\begin{array}{l}\text { Administrative, executive, and managerial } \\
\text { workers }\end{array}$ & $1 \cdot 39$ & $1.25-1.55$ & $<10^{-8}$ & 1.35 & $1 \cdot 21-1 \cdot 51$ & $10^{-7}$ & 0 \\
\hline 4 & $\begin{array}{l}\text { (32) Other diseases of the respiratory system } \\
\text { Farmers, fishermen, hunters, and timbergetters } \\
\text { (33) Diseases of oral cavity, salivary glands, } \\
\text { and jaws }\end{array}$ & $1 \cdot 52$ & $1 \cdot 35-1 \cdot 70$ & $<10^{-8}$ & $1 \cdot 37$ & $1.22-1.54$ & $10^{-7}$ & 2 \\
\hline 1 & $\begin{array}{l}\text { Administrative, executive, and managerial } \\
\text { workers }\end{array}$ & 1.47 & $1.28-1.69$ & $10^{-7}$ & 1.42 & $1.23-1.64$ & $10^{-6}$ & 0 \\
\hline 2 & $\begin{array}{l}\text { Clerical workers } \\
\text { (34) Diseases of other parts of the digestive } \\
\text { system }\end{array}$ & $1 \cdot 38$ & $1.20-1.59$ & $10^{-4}$ & 1.71 & $1.48-1.98$ & $<10^{-8}$ & 0 \\
\hline 1 & $\begin{array}{l}\text { Ádministrative, executive, and managerial } \\
\text { workers }\end{array}$ & $1 \cdot 17$ & $1 \cdot 10-1 \cdot 24$ & $10^{-6}$ & $1 \cdot 11$ & $1.04-1 \cdot 19$ & 0.004 & 2 \\
\hline 9 & $\begin{array}{l}\text { Service, sport, and recreation workers } \\
\text { (36) Diseases of the male genital organs }\end{array}$ & $1 \cdot 16$ & $1.07-1.27$ & 0.002 & $1 \cdot 12$ & $1.02-1.22$ & 0.020 & 2 \\
\hline 1 & $\begin{array}{l}\text { Administrative, executive, and managerial } \\
\text { workers } \\
\text { (47) Fractures }\end{array}$ & $1 \cdot 24$ & $1.09-1.42$ & 0.003 & $1 \cdot 16$ & $1 \cdot 01-1 \cdot 32$ & 0.048 & 1 \\
\hline \multirow[t]{2}{*}{$\begin{array}{l}4 \\
5 \\
6 \\
7 / 8\end{array}$} & $\begin{array}{l}\text { Farmers, fishermen, hunters, and timbergetters } \\
\text { Miners, quarrymen, and related workers } \\
\text { Workers in transport and communications } \\
\text { Craftsmen, production process workers, }\end{array}$ & $\begin{array}{l}1 \cdot 46 \\
1.60 \\
1 \cdot 38\end{array}$ & $\begin{array}{l}1.35-1.59 \\
1.37-1.86 \\
1.24-1.52\end{array}$ & $\begin{array}{r}<10^{-8} \\
10^{-8} \\
<10^{-8}\end{array}$ & $\begin{array}{l}1 \cdot 25 \\
1 \cdot 56 \\
1 \cdot 12\end{array}$ & $\begin{array}{l}1 \cdot 15-1 \cdot 36 \\
1 \cdot 33-1 \cdot 84 \\
1.01-1 \cdot 24\end{array}$ & $\begin{array}{l}10^{-7} \\
10^{-7} \\
0.050\end{array}$ & $\begin{array}{l}6 \\
6 \\
6\end{array}$ \\
\hline & $\begin{array}{l}\text { and labourers } \\
\text { (48) Dislocations, sprains, and strains }\end{array}$ & $1 \cdot 22$ & $1 \cdot 15-1 \cdot 29$ & $<10^{-8}$ & $1 \cdot 14$ & $1 \cdot 08-1 \cdot 21$ & $10^{-6}$ & 3 \\
\hline 4 & $\begin{array}{l}\text { Farmers, fishermen, hunters, and timbergetters } \\
\text { (49) Intracranial and internal injuries including } \\
\text { nerves }\end{array}$ & $1 \cdot 53$ & $1.38-1.71$ & $<10^{-8}$ & $1 \cdot 31$ & $1 \cdot 17-1 \cdot 46$ & $10^{-6}$ & 6 \\
\hline \multirow{2}{*}{$\begin{array}{l}4 \\
5 \\
7 / 8\end{array}$} & $\begin{array}{l}\text { Farmers, fishermen, hunters, and timbergetters } \\
\text { Miners, quarrymen, and related workers }\end{array}$ & $\begin{array}{l}1.68 \\
1.94\end{array}$ & $\begin{array}{l}1 \cdot 53-1 \cdot 84 \\
1 \cdot 62-2 \cdot 31\end{array}$ & $\begin{array}{l}<10^{-8} \\
<10^{-8}\end{array}$ & $\begin{array}{l}1.44 \\
1.90\end{array}$ & $\begin{array}{l}1 \cdot 31-1 \cdot 59 \\
1 \cdot 58-2 \cdot 28\end{array}$ & $\begin{array}{l}<10^{-8} \\
<10^{-8}\end{array}$ & $\begin{array}{l}2 \\
6\end{array}$ \\
\hline & $\begin{array}{l}\text { Craftsmen, production process workers, } \\
\text { and labourers }\end{array}$ & $1 \cdot 52$ & $1.42-1.63$ & $<10^{-8}$ & 1.45 & $1 \cdot 35-1 \cdot 55$ & $<10^{-8}$ & 2 \\
\hline
\end{tabular}




\begin{tabular}{|c|c|c|c|c|c|c|c|c|}
\hline \multirow{2}{*}{\multicolumn{2}{|c|}{ Major occupational group }} & \multicolumn{3}{|c|}{ Rate ratio } & \multicolumn{3}{|c|}{ Odds ratio } & \multirow{2}{*}{$\begin{array}{l}\text { Credibility } \\
\text { score } \\
\text { (Range 0-6) }\end{array}$} \\
\hline & & $\overline{E s t}$ & $95 \% \mathrm{CI}$ & Null p value & $\overline{E s t}$ & $95 \% \mathrm{CI}$ & Null $p$ value & \\
\hline \multirow{2}{*}{$\begin{array}{l}5 \\
6 \\
7 / 8\end{array}$} & \multirow{2}{*}{$\begin{array}{l}\text { (50) Open wounds } \\
\text { Miners, quarrymen, and related workers } \\
\text { Workers in transport and communications } \\
\text { Craftsmen, production process workers, } \\
\text { and labourers } \\
\text { (51) Effects of foreign body entering through } \\
\text { orifice }\end{array}$} & $\begin{array}{l}1.42 \\
1.56\end{array}$ & $\begin{array}{l}1 \cdot 15-1 \cdot 77 \\
1 \cdot 37-1 \cdot 78\end{array}$ & $<10^{-8}$ & $\begin{array}{l}1.42 \\
1.30\end{array}$ & $\begin{array}{l}1 \cdot 13-1 \cdot 78 \\
1 \cdot 13-1 \cdot 48\end{array}$ & $\begin{array}{l}0.007 \\
0.001\end{array}$ & $\begin{array}{l}6 \\
6\end{array}$ \\
\hline & & $1 \cdot 25$ & $1 \cdot 16-1 \cdot 35$ & $<10^{-8}$ & $1 \cdot 17$ & $1 \cdot 09-1 \cdot 27$ & $10^{-5}$ & 4 \\
\hline \multirow{3}{*}{$\begin{array}{c}4 \\
(52) \\
4 \\
7 / 8\end{array}$} & $\begin{array}{l}\text { Farmers, fishermen, hunters, and timbergetters } \\
\text { Burns }\end{array}$ & $2 \cdot 96$ & $2 \cdot 17-4 \cdot 06$ & $<10^{-8}$ & $2 \cdot 61$ & $1.91-3.58$ & $10^{-8}$ & 2 \\
\hline & Farmers, fishermen, hunters, and timbergetters & $2 \cdot 37$ & $1.99-2.82$ & $<10^{-8}$ & 2.00 & $1.67-2.38$ & $<10^{-8}$ & 4 \\
\hline & $\begin{array}{l}\text { and labourers } \\
\text { (53) Poisoning and toxic effects }\end{array}$ & $1 \cdot 70$ & $1.47-1.95$ & $<10^{-8}$ & 1.58 & $1 \cdot 37-1 \cdot 82$ & $<10^{-8}$ & 3 \\
\hline $7 / 8$ & $\begin{array}{l}\text { Craftsmen, production process workers, } \\
\text { and labourers } \\
\text { (54) Complications of medical and surgical care }\end{array}$ & $1 \cdot 24$ & $1.09-1.41$ & 0.001 & $1 \cdot 16$ & $1 \cdot 02-1 \cdot 32$ & 0.028 & 3 \\
\hline $7 / 8$ & $\begin{array}{l}\text { Craftsmen, production process workers, } \\
\text { and labourers } \\
\text { (55) Other injuries, early complications of trauma }\end{array}$ & $1 \cdot 36$ & $1 \cdot 17-1 \cdot 58$ & $10^{-5}$ & $1 \cdot 29$ & $1 \cdot 11-1 \cdot 50$ & $10^{-4}$ & 0 \\
\hline \multirow[t]{2}{*}{$\begin{array}{l}4 \\
5 \\
6 \\
7 / 8\end{array}$} & $\begin{array}{l}\text { Farmers, fishermen, hunters, and timbergetters } \\
\text { Miners, quarrymen, and related workers } \\
\text { Workers in transport and communications } \\
\text { Craftsmen, production process workers, }\end{array}$ & $\begin{array}{l}2.06 \\
2.76 \\
1.53\end{array}$ & $\begin{array}{l}1 \cdot 89-2 \cdot 25 \\
2 \cdot 38-3 \cdot 20 \\
1 \cdot 37-1 \cdot 72\end{array}$ & $\begin{array}{l}<10^{-8} \\
<10^{-8} \\
<10^{-8}\end{array}$ & $\begin{array}{l}1 \cdot 78 \\
2 \cdot 73 \\
1 \cdot 25\end{array}$ & $\begin{array}{l}1 \cdot 62-1 \cdot 95 \\
2 \cdot 33-3 \cdot 18 \\
1 \cdot 11-1 \cdot 40\end{array}$ & $\begin{aligned}<10^{-8} \\
<10^{-8} \\
0.001\end{aligned}$ & $\begin{array}{l}2 \\
4 \\
6\end{array}$ \\
\hline & $\begin{array}{l}\text { and labourers } \\
(56) \text { Late effects of injuries, of poisonings, of toxic } \\
\text { effects, and of other external causes }\end{array}$ & $c^{1 \cdot 19}$ & $1 \cdot 11-1 \cdot 28$ & $10^{-6}$ & $1 \cdot 12$ & $1.04-1.20$ & 0.002 & 5 \\
\hline $7 / 8$ & $\begin{array}{l}\text { Craftsmen, production process workers, } \\
\text { and labourers } \\
\text { (E47) Transport accidents }\end{array}$ & 1.68 & $1 \cdot 28-2 \cdot 21$ & $10^{-4}$ & $1 \cdot 58$ & $1 \cdot 20-2 \cdot 08$ & 0.001 & 3 \\
\hline $\begin{array}{l}5 \\
6 \\
7 / 8\end{array}$ & $\begin{array}{l}\text { Miners, quarrymen, and related workers } \\
\text { Workers in transport and communications } \\
\text { Craftsmen, production process workers,... }\end{array}$ & $\begin{array}{l}1.52 \\
1.83\end{array}$ & $\begin{array}{l}1.27-1.82 \\
1.66-2.04\end{array}$ & $\begin{array}{r}10^{-5} \\
<10^{-8}\end{array}$ & $\begin{array}{l}1.59 \\
1.53\end{array}$ & $\begin{array}{l}1.32-1.93 \\
1.37-1.71\end{array}$ & $\begin{array}{r}10^{-5} \\
<10^{-8}\end{array}$ & $\begin{array}{l}5 \\
6\end{array}$ \\
\hline 9 & $\begin{array}{l}\text { and labourers } \\
\text { Service, sport, and recreation workers } \\
\text { (E48) Accidental poisoning }\end{array}$ & $\begin{array}{l}1 \cdot 16 \\
1 \cdot 20\end{array}$ & $\begin{array}{l}1.09-1 \cdot 24 \\
1.05-1 \cdot 37\end{array}$ & $\begin{array}{l}10^{-6} \\
0.017\end{array}$ & $\begin{array}{l}1.08 \\
1.22\end{array}$ & $\begin{array}{l}1 \cdot 01-1 \cdot 16 \\
1.06-1 \cdot 41\end{array}$ & $\begin{array}{l}0.025 \\
0.009\end{array}$ & $\begin{array}{l}1 \\
1\end{array}$ \\
\hline 4 & $\begin{array}{l}\text { Farmers, fishermen, hunters, and timbergetters } \\
\text { (E50) Accidental falls }\end{array}$ & $2 \cdot 38$ & $1.69-3.35$ & $10^{-6}$ & 2.02 & $1.43-2.85$ & $10^{-4}$ & 4 \\
\hline $7 / 8$ & $\begin{array}{l}\text { Craftsmen, production process workers, } \\
\text { and labourers } \\
\text { (E51) Accidents caused by fire and flames }\end{array}$ & $1 \cdot 33$ & $1 \cdot 20-1.46$ & $10^{-8}$ & 1.25 & $1 \cdot 14-1 \cdot 38$ & $10^{-6}$ & 5 \\
\hline \multirow[t]{2}{*}{$\stackrel{4}{7 / 8}$} & $\begin{array}{l}\text { Farmers, fishermen, hunters, and timbergetters } \\
\text { Craftsmen, production process workers. }\end{array}$ & $3 \cdot 25$ & $2 \cdot 48-4 \cdot 27$ & $<10^{-8}$ & $2 \cdot 73$ & $2.08-3.59$ & $<10^{-8}$ & 5 \\
\hline & $\begin{array}{l}\text { and labourers } \\
\text { (E52) Other accidents including late effects }\end{array}$ & 1.53 & $1 \cdot 20-1.94$ & $10^{-4}$ & 1.42 & $1 \cdot 12-1 \cdot 80$ & 0.004 & 1 \\
\hline \multirow[t]{2}{*}{$\begin{array}{l}4 \\
5 \\
7 / 8\end{array}$} & $\begin{array}{l}\text { Farmers, fishermen, hunters, and timbergetters } \\
\text { Miners, quarrymen, and related workers } \\
\text { Craftsmen, production process workers, }\end{array}$ & $\begin{array}{l}1 \cdot 78 \\
1.80\end{array}$ & $\begin{array}{l}1.70-1.86 \\
1.65-1.97\end{array}$ & $\begin{array}{l}<10^{-8} \\
<10^{-8}\end{array}$ & $\begin{array}{l}1.66 \\
1.93\end{array}$ & $\begin{array}{l}1.58-1.75 \\
1.74-2.15\end{array}$ & $\begin{array}{l}<10^{-8} \\
<10^{-8}\end{array}$ & $\begin{array}{l}5 \\
5\end{array}$ \\
\hline & $\begin{array}{l}\text { and labourers } \\
\text { (E54) Suicide and self inflicted injury }\end{array}$ & $1 \cdot 26$ & $1 \cdot 22-1 \cdot 31$ & $<10^{-8}$ & 1.22 & $1 \cdot 18-1 \cdot 27$ & $<10^{-8}$ & 4 \\
\hline $7 / 8$ & $\begin{array}{l}\text { Craftsmen, production process workers, } \\
\text { and labourers } \\
\text { (E55) Homicide and injury purposely inflicted by } \\
\text { other persons }\end{array}$ & $1 \cdot 52$ & $1 \cdot 29-1 \cdot 79$ & $10^{-6}$ & $1 \cdot 41$ & $1.20-1.66$ & $10^{-5}$ & 1 \\
\hline $7 / 8$ & $\begin{array}{l}\text { Craftsmen, production process workers, } \\
\text { and labourers } \\
\text { (VO) Other reasons for contact with health } \\
\text { services }\end{array}$ & $1 \cdot 59$ & $1.38-1.84$ & $<10^{-8}$ & 1.48 & $1 \cdot 28-1 \cdot 70$ & $10^{-8}$ & 1 \\
\hline 0 & Professional, technical, and related workers & $1 \cdot 30$ & $1 \cdot 22-1 \cdot 38$ & $<10^{-8}$ & $1 \cdot 29$ & $1 \cdot 21-1 \cdot 37$ & $<10^{-8}$ & 2 \\
\hline
\end{tabular}

*Score unavailable due to clerical error.

selection of referent events that met the criterion of no association with the exposure. ${ }^{5}$ In this study the sheer bulk of data precluded such a considered judgment for each of the hundreds of associations that were examined. For referent events we have, in all instances, used the total numbers of hospital discharges in the exposed and non-exposed groups due to all conditions other than the particular ICD-9 chapter or basic tabulation list item under study. Compared with the $R R$, the $O R$ is less susceptible to selection bias due to healthy or unhealthy workers accumulated in certain occupational groups, or a variable propensity of different occupational groups for health care use. ${ }^{67}$ The disadvantage of the $O R$ is that its validity is crucially dependent on the absence of an association of the referent events with the occupational exposure 
under study. Thus the $\mathrm{OR}$ and $\mathrm{RR}$ are subject to different potential sources of bias. Both measures are preferable to the SMR (standardised mortality or morbidity ratio) and PMR which, being indirectly standardised parameters, have inherent problems when two or more are compared. ${ }^{8}$

CONSUlTATIVE PANEL

The impact of any one epidemiological study on scientific knowledge is determined by the extent to which it modifies existing views about causal associations. Thus the description of an isolated result as "significant" or not, without taking into account existing knowledge, bears little resemblance to how scientific knowledge is actually advanced. Formal Bayesian statistical methods for combining study information with prior credibility to obtain an updated view are, as yet, insufficiently developed for general use. Therefore, in the belief that the Bayesian outlook is essentially correct, we have incorporated a limited version of the approach into the analysis.

A consultative panel, BKA, JCM, and PP-S, were given the task of scoring the prior credibility of each empirical association in which the $p$ values of both the $R R$ and $O R$ were less than $0 \cdot 05$. Because the criterion entailed a departure of both the RR and OR from unity, the potential for bias as an explanation for the findings was reduced.

Each member of the panel made an independent evaluation of the prior credibility of associations based on his existing knowledge and insight and scored the association according to the following three step scale:

Score

Interpretation

0 No prior credibility: the referee is unaware of any possible mechanism for the association, nor does the referee regard the association as raising any suspicion that it might be real. The referee considers that the most likely explanation is chance.

1 Limited prior credibility: the referee is unaware of a detailed possible mechanism for the association, but neverthelesss regards it as raising suspicion. The referee considers that with further study, the association might prove not to be due to chance.

2 High prior credibility: the referee is aware of a possible mechanism for the association and considers that it is likely to be real rather than due to chance.

These scores were assigned without knowledge of the basis for selection of the associations to be scored, and some associations with high $\mathrm{p}$ values were included in the list to minimise any effect that a perception of the results might otherwise have had on the "prior" judgment.

The three credibility scores obtained for each association were summed to give a final score on a scale of zero to six, where zero means no prior credibility and six means very high prior credibility. These summary indices of prior credibility have been included in tables 2 and 3. By studying the credibility scores, a reader unfamiliar with occupational health may obtain some appreciation of which empirical associations were more likely to have arisen by chance.

\section{Results and discussion}

There were 101548 hospital discharges among employed men in the two year period 1981-2. Table 4 lists the number of hospital discharges for the ICD-9 chapters in order of frequency. Conditions coded to chapter XVII, injury and poisoning, were the most common cause of entering hospital, accounting for $20 \cdot 2 \%$. Poisonings accounted for only $0.9 \%$ and so the vast majority of these admissions were the result of injury. The second most common cause was diseases of the musculoskeletal system and connective tissue (12.6\% of admissions).

The 101548 hospital discharges among employed men gave a crude discharge rate of 150 per 1000 person-years. In general, the age specific rates rose with age, except that those due to injury and poisonings were the highest in men aged 15-24 and declined at older ages.

Table 4 Number of hospital discharges of employed men in Western Australia in 1981-2 for the ICD-9 chapters in order of frequency

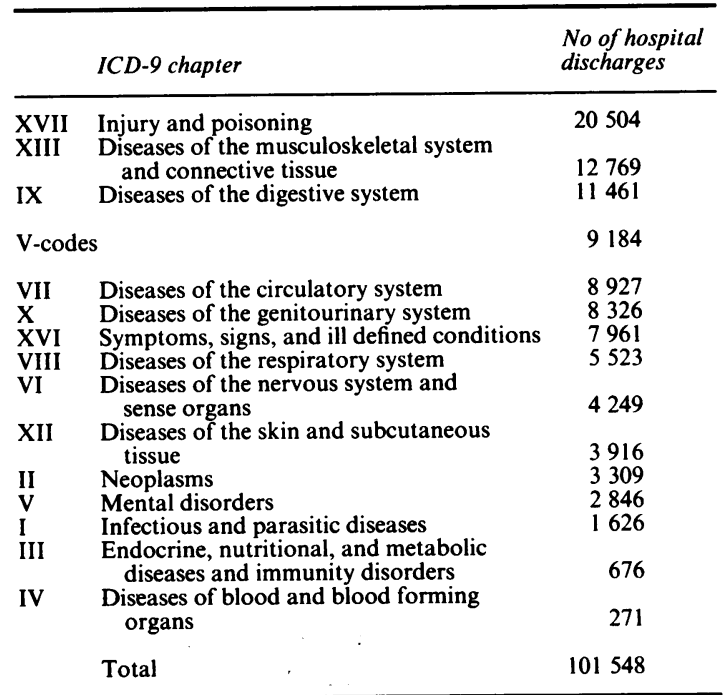


Table 5 Age adjusted hospital discharge rate ratios for Western Australian men aged 15-64 in 1981-2 according to major occupational group

\begin{tabular}{|c|c|c|c|c|}
\hline & \multirow[b]{2}{*}{ Major occupational group } & \multicolumn{3}{|c|}{ Rate ratio } \\
\hline & & $\overline{E s t}$ & $95 \% C I$ & Null $p$ value \\
\hline $\begin{array}{l}0 \\
1 \\
2 \\
3 \\
4 \\
5 \\
6 \\
7 / 8 \\
9 \\
10\end{array}$ & $\begin{array}{l}\text { Professional, technical, and related workers } \\
\text { Administrative, executive, and managerial workers } \\
\text { Clerical workers } \\
\text { Sales workers } \\
\text { Farmers, fishermen, hunters, and timbergetters } \\
\text { Miners, quarrymen, and related workers } \\
\text { Workers in transport and communications } \\
\text { Craftsmen, production process workers, and labourers, nec } \\
\text { Service, sport, and recreation workers } \\
\text { Members of armed services }\end{array}$ & $\begin{array}{l}0.78 \\
1.06 \\
0.82 \\
0.77 \\
1.13 \\
1.16 \\
1.25 \\
1.05 \\
1.05 \\
1.32\end{array}$ & $\begin{array}{l}0.76-0.80 \\
1 \cdot 04-1 \cdot 08 \\
0 \cdot 80-0 \cdot 84 \\
0 \cdot 75-0.80 \\
1 \cdot 11-1 \cdot 16 \\
1 \cdot 11-1 \cdot 21 \\
1 \cdot 23-1.28 \\
1.04-1.06 \\
1 \cdot 02-1.08 \\
1 \cdot 24-1.41\end{array}$ & $\begin{aligned} &< 10^{-8} \\
& 10^{-7} \\
&<10^{-8} \\
&<10^{-8} \\
&<10^{-8} \\
&<10^{-8} \\
&<10^{-8} \\
&<10^{-8} \\
& 0.002 \\
&<10^{-8}\end{aligned}$ \\
\hline
\end{tabular}

nec $=$ Not elsewhere classified.

Table 5 shows the RR estimates based on the total discharges that occurred in each major occupational group. (OR calculations were not possible for total discharges.) In every case the $p$ value associated with the RR comparing each occupational group to all others was less than $0 \cdot 05$. In some instances this was despite a minor departure of the rate ratio estimate from unity, reflecting the high level of statistical power obtained from large numbers of hospital admissions. Members of the armed services had the greatest excess of admissions ( $R R=1 \cdot 32$ ), followed by workers in transport and communications $(R R=1 \cdot 25)$. Farmers, fishermen, hunters, and timbergetters ( $R \mathbf{R}=$ $1 \cdot 13$ ), as well as miners, quarrymen, and related workers $(R R=1 \cdot 16)$ experienced intermediate levels of admissions to hospital, whereas professional, technical, and related workers $(R R=0.78)$, clerical workers $(R R=0.82)$, and sales workers $(R R=0.77)$ were admitted at low rates compared with other groups.

If the age specific hospital morbidity rates in sales workers were to have applied to the total population of working men in 1981-2, then the expected number of hospital discharges would have been 76218 . It may be implied, therefore, that if all men in the workforce had experienced the same rate of admission as sales workers there would have been around 25330 fewer hospital admissions over the two year period.

Table 2 presents the empirical results and the aggregate credibility scores allotted by the consultative panel for the analysis by ICD-9 chapter. The table is restricted to associations referred to the consultative panel-that is, those in which the RR or OR had $p$ values of less than 0.05 . Table 3 shows the results of the analysis by ICD-9 basic tabulation list, again restricted to associations referred to the consultative panel. By cross referencing these results with those in table 2, more specific detail of the nature of associations may be obtained.

We divided the empirical associations into four categories:
Strong associations-where the $\mathrm{p}$ values for the $\mathrm{OR}$ and RR were $<0.05$ and the credibility score was 5 or 6.

Possible associations-where the $\mathrm{p}$ values for the OR and RR were $<0.05$ and the credibility score was 2,3 , or 4 .

Associations with low credibility - where the p values for the odds ratio and rate ratio were $<0.05$ but the credibility score was 0 or 1 .

Omissions - that is, empirical associations that on the basis of prior knowledge would have been expected to be found but were not.

\section{STRONG ASSOCIATIONS}

All the strong associations were observed among items relating to injury and poisoning or diseases of the musculoskeletal system and connective tissue (see tables 2 and 3 ).

Four major occupational groups were frequently associated with injury. These were farmers, fishermen, hunters, and timbergetters; miners, quarrymen, and related workers; workers in transport and communications; and craftsmen, production process workers, and labourers. These associations received high credibility scores, it being likely that men in these occupations were more at risk of trauma.

Associations were shown between musculoskeletal and connective tissue conditions and workers in transport and communications, and craftsmen, production process workers, and labourers. Both were ascribed a high credibility score. Drivers could suffer from dorsopathies due to factors such as vibration and lack of ergonomic design of drivers' seats and vehicle controls.' Dorsopathies and other musculoskeletal problems may be common among manual workers because of heavy lifting or repetitive injury..$^{910}$

POSSIBLE ASSOCIATIONS

There was an association between infectious and parasitic diseases and the occupational group of farmers, fishermen, hunters, and timbergetters. This 
may possibly have been due to zoonoses or to substandard living conditions while in the field. ${ }^{11}$ Indeed, the basic tabulation list analysis showed an association between this occupational group and intestinal infectious diseases, although the panel gave this a credibility score of only 1 . Service, sport, and recreation work was also associated with infectious and parasitic diseases. In the more detailed analysis, however, this group of occupations was associated with viral diseases with a credibility score of 0 . That said, it is possible that service workers with their high level of contact with other people may be at increased risk of contacting, through person to person transmission, a viral infection.

There was an association between neoplasms and professional, technical, and related workers. The basic tabulation list analysis showed an underlying association with benign neoplasms, which was possibly due to a greater access of professional workers to investigation and health care services. Another underlying association was with malignant neoplasms of bone, connective tissue, skin, and breast. The relation between malignant melanoma and indoor work may explain this finding as clerical work was also associated with this category of cancers. ${ }^{12}$ The association between neoplasms and members of the armed services did not appear to be due to increased consumption of alcohol and tobacco, as no excess of respiratory tract or gastrointestinal tract malignancies was observed.

Diseases of the circulatory system were associated with professional, technical, and related workers, and administrative, executive, and managerial workers, with credibility scores of 2 and 3 , respectively. Risk factors prevalent in people in these occupations may include stress, sedentary work, and overnutrition. ${ }^{13}$ Clerical workers had an increased hospital admission rate from ischaemic heart disease and other diseases of the circulatory system, also perhaps because of the sedentary nature of their occupation. ${ }^{13}$ Moreover, some jobs designated as "clerk" may have been, in fact, administrative or managerial in nature.

Diseases of the respiratory system were associated with administrative, executive, and managerial work with a credibility score of 2 . The main disease component of this association was diseases of the upper respiratory tract for which no ready explanation can be offered. Farmers, fishermen, hunters, and timbergetters had an increased rate of admission for other diseases of the respiratory system, with a credibility score of 2. Occupational asthma and extrinsic allergic alveolitis might have contributed to this association. ${ }^{14}$

Another "possible" association was between administrative, executive, and managerial workers and diseases of the digestive system. Discharges coded to "other diseases of the digestive system"-that is, excluding oral cavity, salivary glands, and jaw-formed the basis of this association. Stress, irregular eating habits, and excess alcohol consumption leading to an increased incidence of peptic ulceration, pancreatitis, and other alcohol related conditions might be some of the underlying causes. ${ }^{1516}$ Diseases of other parts of the digestive system were also associated with service, sport, and recreation workers. The problems described above may also apply in this group, particularly to service workers such as policemen and the fire brigade.

Farmers, fishermen, hunters, and timbergetters had an increased rate of symptoms, signs, and ill defined conditions, with a credibility score of 2 . Possibly, however, this was a spurious relation due to their rural residence and their access to diagnostic services being reduced in comparison with metropolitan residents.

\section{ASSOCIATIONS WITH LOW CREDIBILITY}

Among the chapters there were three associations and in the basic tabulation list 22 associations that were ascribed low credibility by the panel - that is, a score of 0 or 1.

Whereas most of these associations were probably due to chance, a few might be tenable. For example, the association between administrative, executive, and managerial work and diseases of the upper respiratory tract may be contributed to by the "tight building syndrome." The tight (or sick) building syndrome is the clustering of complaints of ill health that occurs in workplaces, usually in office blocks, that are air conditioned. Eye and respiratory tract symptoms and headaches are among the most frequently reported. The cause of these symptoms remains obscure. ${ }^{17}$

\section{OMISSIONS}

Several associations were expected but not found. For an association to be manifest in this type of analysis, the disease outcome must be a relatively common cause of admission to hospital, and this has been recognised in discussion of omissions. For example, an increased rate of non-melanocytic skin cancer in farmers, fishermen, hunters, and timbergetters ${ }^{18}$ may not have appeared in our data because this type of skin cancer in Australia rarely leads to admission. Although asbestos miners suffer an increased rate of cancers of the lung and pleura, the numbers of such workers in Western Australia are probably too small for an effect to be apparent.

No association was observed between craftsmen, production process workers, and labourers, and diseases of the skin and subcutaneous tissue, despite the potential for industrial dermatitis in this group. ${ }^{13}$ The omission is probably explained by a low admission rate for industrial dermatitis.

Perhaps the most surprising omission is of an 
association between respiratory disorders and miners, quarrymen, and related workers. ${ }^{14}$ It may be that workers in this group with respiratory diseases give up their jobs if the disease is severe enough to warrant hospital admission. In other words, the omission of this association may be a manifestation of the healthy worker effect which was beyond the ability of our study methods to control.

\section{CONCLUDING COMMENT}

An individual's occupation has important effects on health. These effects may be divided into intrinsic factors, which include the type of work undertaken and exposures to potentially harmful agents such as chemicals or dangerous machinery, and extrinsic factors such as lifestyle, general health, smoking, alcohol consumption, recreational pursuits, and place of residence. ${ }^{19}$ Men in higher status occupations are known to have a lower total death rate than men in lower status occupations, a difference that is due to variations in both intrinsic and extrinsic factors. ${ }^{67}$ Likewise, both types of occupation related factors must be considered in interpretation of the results of this study.

Taylor et al in their study of occupation and mortality in Australia concluded that men in major occupational groups 5, 6, 7/8, and 9 (the "blue collar" workers) had higher mortality rates than men in groups $0,1,2$, and 3 (the "white collar" workers) for "all causes" and for selected causes of death such as ischaemic heart disease, cerebrovascular disease, malignant neoplasia, accidents, and respiratory diseases. ${ }^{20}$ Whereas the same conclusion about hospital morbidity could be drawn for accidents from our data, higher rates for blue collar workers in the other selected causes mentioned above were not found for hospital morbidity. To ascertain whether the rates for all causes of hospital morbidity were higher in blue collar than in white collar workers, total age standardised rates for the two groups of workers were calculated. The rate for blue collar workers was 161.74 $(\mathrm{SE}+0.64)$ and for white collar workers 132.84 (SE + $0.78)$, giving a rate difference of $28.90(95 \% \mathrm{CI}, 26.92$ 30.88) hospital discharges per 1000 person-years. Injury and poisoning made the greatest contribution to the difference between the two groups of workers.

This study has demonstrated that substantial differences exist in the rates at which different occupational groups are admitted to hospital, sales workers having the fewest and armed services personnel the most frequent admissions to hospital. In the absence of this variation - that is, if all occupational groups had hospital morbidity equal to that in sales workers, it is estimated that in Western Australia in 1981-2 there would have been 12665 fewer hospital admissions per year or $24.9 \%$ of the total in working men.

We thank Mr Beress Brooks who provided data from the Hospital Morbidity Data System, the Australian Bureau of Statistics for population data, and Miss Helen Lammle for secretarial support.

\section{References}

1 Waddell VP, Holman CDJ. A population-based study of hospital morbidity by occupation in Western Australian males aged 15-64 years; 1981-82. Perth: Health Department of Western Australia, 1985.

2 Kleinbaum DG, Kupper LL, Morgenstern H. Epidemiologic research principles and quantitative methods. Belmont, California: Wadsworth Inc, 1982.

3 Rothman KJ. Modern epidemiology. Boston: Little, Brown \& Co, 1986.

4 Miettinen OS, Wang JD. An alternative to the proportionate mortality ratio. Am J Epidemiol 1981;114:144-8.

5 Wang JD, Miettinen OS. The mortality odds ratio (MOR) in occupational mortality studies - selection of reference occupation(s) and reference cause(s) of death. Ann Acad Med 1984;13:312-6.

6 Morris JN. Social inequalities undiminished. Lancet 1979;i:87-90.

7 Wilson RW, White EL. Changes in morbidity, disability and utilisation differentials between the poor and the nonpoor. Medical Care 1977;15:636-47.

8 Miettinen OS. Standardisation of risk ratios. Am J Epidemiol 1972;96:383-8.

9 Grandjean E. Fitting the task to the man: an ergonomic approach. London: Taylor and Francis Ltd, 1982:41-62, 322-3.

10 National Health and Medical Research Council. Back injury at work. Approved occupational health guide. Canberra: NH \& MRC, 1981.

11 National Health and Medical Research Council. Zoonoses. Approved occupational health guide. Canberra: NH \& MRC, 1981.

12 Holman CDJ, Mulroney CD, Armstrong BK. Epidemiology of pre-invasive and invasive malignant melanoma in Western Australia. Int J Cancer 1980;25:317-23.

13 Fine LJ. Occupational heart disease. In: Rom W, ed. Environmental and occupational medicine. Boston: Little, Brown and Co, 1983:359-65.

14 Parkes W. Occupational lung disease. London: Butterworths, 1982:359-453.

15 Richardson CT. Peptic ulcer. In: Wyngaarden JB, Smith LH, eds. Cecil textbook of medicine. Philadelphia: WB Saunders and Co, 1985:681-96.

16 Kissin B. Alcohol abuse and alcohol-related illness. In: Wyngaarden JB, Smith LH, eds. Cecil textbook of medicine. Philadelphia: WB Saunders and Co, 1985:54-5.

17 Finnegan MJ, Pickering CAC, Burge PS. The sick building syndrome: prevalence studies. Br Med J 1984;289:1573-5.

18 Silverstone $\mathrm{H}$, Searle JH. The epidemiology of skin cancer in Queensland: the influence of phenotype and environment. $\boldsymbol{B r} J$ Cancer 1970;24:235-52.

19 Office of Population Censuses and Surveys. Occupational mortality, decennial supplement, 1970-72. England and Wales. London: OPCS, 1978.

20 Taylor R, Herrman H, Preston G. Occupation and mortality in Australia, working age males, 1975-77. Melbourne: Health Commission of Victoria and Department of Social and Preventive Medicine, Monash University, 1983. 